\title{
ASYMMETRIC MAXIMAL IDEALS IN $M(G)$
}

\author{
BY
}

\section{SADAHIRO SAEKI}

\begin{abstract}
Let $G$ be a nondiscrete LCA group, $M(G)$ the measure algebra of $G$, and $M_{0}(G)$ the closed ideal of those measures in $M(G)$ whose Fourier transforms vanish at infinity. Let $\Delta_{G}, \Sigma_{G}$ and $\Delta_{0}$ be the spectrum of $M(G)$, the set of all symmetric elements of $\Delta_{G}$, and the spectrum of $M_{0}(G)$, respectively. In this paper this is shown: Let $\Phi$ be a separable subset of $M(G)$. Then there exist a probability measure $\tau$ in $M_{0}(G)$ and a compact subset $X$ of $\Delta_{0} \mid \Sigma_{G}$ such that for each $|c| \leqslant 1$ and each $\nu \in \Phi \operatorname{Card}\{f \in X: \hat{\tau}(f)=c$ and $|\hat{\nu}(f)|=r(\nu)\} \geqslant 2^{c}$.

Here $r(\nu)=\sup \left\{|\hat{\nu}(f)|: f \in \Delta_{G} \mid \hat{G}\right\}$. As immediate consequences of this result, we have (a) every boundary for $M_{0}(G)$ is a boundary for $M(G)$ (a result due to Brown and Moran), (b) $\Delta_{G} \backslash \Sigma_{G}$ is dense in $\Delta_{G} \backslash \hat{G}$, (c) the set of all peak points for $M(G)$ is $\hat{G}$ if $G$ is $\sigma$-compact and is empty otherwise, and (d) for each $\mu \in$ $M(G)$ the set $\hat{\mu}\left(\Delta_{0} \mid \Sigma_{G}\right)$ contains the topological boundary of $\hat{\mu}\left(\Delta_{G} \backslash \hat{G}\right)$ in the complex plane.
\end{abstract}

Throughout the paper, let $G$ be a nondiscrete locally compact abelian group with dual $\hat{G}, M(G)$ the convolution measure algebra of $G$, and $M_{0}(G)$ the ideal in $M(G)$ which consists of all measures with Fourier transforms vanishing at infinity. As is well known, we then have $L^{1}(G)=M_{a}(G) \subset M_{0}(G) \subset M_{c}(G)$. Let $\Delta_{G}$ denote the spectrum of $M(G)$, i.e., the space of all nonzero complex homomorphisms of $M(G)$, and let $\hat{\mu}$ denote the Gelfand transform of $\mu \in M(G)$. We define

$$
\begin{aligned}
& \Delta_{0}=\left\{f \in \Delta_{G}: \hat{\sigma}(f) \neq 0 \text { for some } \sigma \in M_{0}(G)\right\}, \\
& \Sigma_{G}=\left\{f \in \Delta_{G}: f\left(\sigma^{*}\right)=\overline{f(\sigma)} \text { for all } \sigma \in M(G)\right\},
\end{aligned}
$$
where $\sigma^{*}(E)=\overline{\sigma(-E)}$ for all Borel sets $E$ in $G$ and $f(\sigma)=\hat{\sigma}(f)$. Then $\Delta_{0}$ is open (in $\Delta_{G}$ ), $\Sigma_{G}$ is closed, and $\hat{G} \subset \Delta_{0} \cap \Sigma_{G}$. Moreover, $\Delta_{0}$ may be identified with the spectrum of $M_{0}(G)$, since $M_{0}(G)$ is an ideal in $M(G)$.

It is shown in [11] that given $\mu \in M_{c}(G)$, there exist fairly many elements $f \in \Delta_{G}$ such that $M_{a}(G)+L^{1}(\mu) \subset \operatorname{Ker}(f)$ but $M_{0}(G) \not \subset \operatorname{Ker}(f)$. In fact, it is not difficult to improve Theorem 2 of [11] as follows.

THEOREM A. Let $0 \neq \lambda \in M_{0}(G), \mu \in M_{c}(G)$, and $H$ a subgroup of $G$ which is a $G_{\delta}$-set. Then there exists a probability measure $\sigma=\tau * \tau^{*}$, with $\tau \dot{\epsilon}$

Received by the editors July $11,1975$.

AMS (MOS) subject classifications (1970). Primary 43A10. boundary.

Key words and phrases. LCA group, measure algebra, asymmetric maximal ideal, $M_{0}$ - 
$M_{0}^{+}(\operatorname{supp} \lambda)$, having the following properties:

(i) Given $0 \leqslant r \leqslant 1$, the set of all $f \in \Sigma_{G}$ such that

$$
\hat{\sigma}(f)=r, \quad \operatorname{Ker}(f) \supset L^{1}(\mu), \quad \text { and } \hat{\nu}(f)=\hat{\nu}(1) \quad \forall \nu \in M_{d}(G)
$$

has cardinality $\geqslant 2^{c}$. Here $c$ denotes the cardinal number of the continuum.

(ii) Given a complex number $c$ of modulus $\leqslant 1$ and $g \in \Delta_{G}$ with $g\left(\delta_{x}\right)=1$ for all $x \in H$, the set of all $f \in \Delta_{G} \backslash \Sigma_{G}$ such that

$$
\hat{\sigma}(f)=c, \quad \operatorname{Ker}(f) \supset L^{1}(\mu), \quad \text { and } \quad \hat{\nu}(f)=\hat{\nu}(g) \quad \forall \nu \in M_{d}(G)
$$

has cardinality $\geqslant 2^{c}$.

For some related results, we refer the reader to Izuchi and Shimizu [8], Saka [12], Shimizu [13], and Williamson [15]. Now let $\mu \in M(G)$ be given, and define

$$
r(\mu)=\sup \left\{|\hat{\mu}(f)|: f \in \Delta_{G} \backslash \hat{G}\right\} .
$$

Since $\Delta_{G} \backslash \hat{G}$ is compact, there exists at least one $f$ in this set such that $|\hat{\mu}(f)|=$ $r(\mu)$. It seems to be a natural problem to ask how many $f$ as above there exist. Our answer is as follows.

Theorem B. Let $\mu \in M(G)$, and $\Phi$ a separable subset of $L^{1}(\mu)$. Then there exist a probability measure $\tau$ in $M_{0}(G)$ and a compact set $X$ in $\Delta_{0} \backslash \Sigma_{G}$ such that

$$
\operatorname{Card}\{f \in X: \hat{\tau}(f)=c \text { and }|\hat{\nu}(f)|=r(\nu)\} \geqslant 2^{c}
$$

for every complex number $c$ of modulus $\leqslant 1$ and every measure $\nu$ in $\left[L^{1}(\mu) \cap\right.$ $\left.M^{+}(G)\right] \cup \Phi$.

Notice that we can set $\Phi=L^{1}(\mu)$ if $G$ is metrizable, since then $L^{1}(\mu)$ is separable. As easy consequences of the last theorem, we have the following results.

\section{COROLLARY 1.}

(a) Every boundary of $M_{0}(G)$ is a boundary of $M(G)$.

(b) The set $\Sigma_{G} \backslash \hat{G}$ is the topological boundary of $\Delta_{G} \backslash \Sigma_{G}$ in $\Delta_{G}$. In other words, $\Delta_{G} \backslash \Sigma_{G}$ is dense in $\Delta_{G} \backslash \hat{G}$.

(c) If $G$ is $\sigma$-compact, then the set $P_{G}$ of all peak points for $M(G)$ is precisely $\hat{G}$. If not, then $P_{G}=\varnothing$.

Corollary 2. For each $\mu \in M(G)$, the set $\hat{\mu}\left(\Delta_{0} \backslash \Sigma_{G}\right)$ contains the topological boundary of $\hat{\mu}\left(\Delta_{G} \backslash \hat{G}\right)$ in the complex plane $\mathbf{C}$. In particular, we have

(a) If Card $\left[\hat{\mu}\left(\Delta_{0} \backslash \Sigma_{G}\right)\right]<c$, then $\hat{\mu}\left(\Delta_{G} \backslash \hat{G}\right)$ is (at most) countable and coincides with $\hat{\mu}\left(\Delta_{0} \backslash \Sigma_{G}\right)$.

(b) If $\hat{\mu}$ is real on $\Delta_{0} \backslash \Sigma_{G}$, then $\hat{\mu}\left(\Delta_{G} \backslash \hat{G}\right)=\hat{\mu}\left(\Delta_{0} \backslash \Sigma_{G}\right)$. 
Notice that Theorem B implies the result of Brown and Moran [2] and Graham [5]: If $\mu \in M(G)$ and $\hat{\mu}=0$ off $\Sigma_{G}$, then $r(\mu)=0$. Part (a) of Corollary 1 is due to Brown and Moran [3]. We also refer to Brown's result in [1]: $\Delta_{0} \cap \Sigma_{G}$ is not entirely contained in the Shilov boundary of $M_{0}(G)$. It may be an interesting problem to ask whether or not we have $\hat{\mu}\left(\Delta_{0} \backslash \Sigma_{G}\right)=\hat{\mu}\left(\Delta_{G} \backslash \hat{G}\right)$ for all $\mu \in M(G)$.

To prove Theorem B, we shall first construct a measure of a certain type (assuming that $G$ is metrizable). The construction of such a measure is almost the same as the corresponding one in [11], and Körner's method [9] plays an important role in our construction.

We now introduce some notation. Let $m_{G}$ denote the Haar measure of $G$, and $\mathbf{Z}$ the group of all integers. For a set $K$ in $G$ and $p \in \mathbf{Z}^{+}$, we define

$$
p K=\left\{x_{1}+\cdots+x_{p}: x_{j} \in K \text { for all } 1 \leqslant j \leqslant p\right\}
$$

if $p \geqslant 1, p K=\{0\}$ if $p=0$, and $(-p) K=-(p K)$. The subgroup of $G$ which the set $K$ generates is denoted by $G p(K)$. We say that a Borel set $K$ is of type $M_{0}$ if $M_{0}(K)=M_{0}(G) \cap M(K)$ is nonzero. Let $q(G)$ denote the supremum of all natural numbers $p$ such that every neighborhood of the identity 0 of $G$ contains an element of order $\geqslant p$. Then it is easy to see that if $q(G)=\infty$, then $G$ is an I-group, and that if $q(G)$ is finite, then $G$ contains an open-and-compact subgroup $H$ such that ord $(x) \leqslant q(G)$ for all $x$ in $H$. A set $K$ in $G$ is called strongly independent if it is independent in the usual sense [10, p. 97] and if all of its elements have order $q(G)$. Finally, we denote by $G p^{\prime}(K)$ the set of all points $x$ of the form $x=k_{1} x_{1}+\cdots+k_{u} x_{u}$, where $u=u_{x}$ is a natural number, $x_{1}, \ldots, x_{u}$ are distinct elements of $K, k_{j} \in Z$ for all $1 \leqslant j \leqslant u$, and $\left|k_{j}\right|=$ 1 for at least one index $j$.

LEMMA 1. Let $\mu_{0} \in M^{+}(G), D$ a compact subset of $G$ with Haar measure zero, and $N$ a natural number. Let also $V_{1}, V_{2}, \ldots, V_{u}$ be nonempty open sets in $G$. Then we can find nonempty open sets $U_{j} \subset V_{j}(1 \leqslant j \leqslant u)$ subject to the following conditions:

(i) If $p_{j} \in \mathrm{Z},\left|p_{j}\right|<q(G)$, and $1 \leqslant \Sigma_{j=1}^{u}\left|p_{j}\right| \leqslant N$, then the set $\Sigma_{j=1}^{u} p_{j} U_{j}$ does not contain $0 \in G$, and

$$
m_{G}\left[D+\sum_{j=1}^{u} p_{j} U_{j}\right]<1 / N .
$$

(ii) If $q_{j} \in \mathbf{Z}, \sum_{j=1}^{u}\left|q_{j}\right| \leqslant N$, and $\left|q_{j}\right|=1$ for at least one index $j$, then

$$
\mu_{0}\left[D+\sum_{j=1}^{u} q_{j} U_{j}\right]<1 / N
$$


Proof. Let $P$ be the set of all $p=\left(p_{1}, \ldots, p_{u}\right) \in Z^{u}$ as in (i). Similarly, let $Q$ be the set of all $q=\left(q_{1}, \ldots, q_{u}\right) \in \mathrm{Z}^{u}$ as in (ii).

The standard Baire category argument $[10,5.2 .3]$ shows that there are points $x_{j} \in V_{j}(1 \leqslant j \leqslant u)$ of order $\geqslant q(G)$ such that $\left\{x_{j}: 1 \leqslant j \leqslant u\right\}$ is independent. Since $P$ is finite and $D$ is a compact set with Haar measure zero, we can find a neighborhood $W$ of $0 \in G$ so that

$$
0 \notin \sum_{1}^{u} p_{j}\left(x_{j}+W\right) \text { and } m_{G}\left[D+\sum_{1}^{u} p_{j}\left(x_{j}+W\right)\right]<1 / N
$$

for all $p \in P$. We may assume that $x_{j}+W \subset V_{j}(1 \leqslant j \leqslant u)$.

Put $E=\left\{x_{j}: 1 \leqslant j \leqslant u\right\}$, and take a compact neighborhood $X$ of $0 \in G$ such that $X+X \subset W$. Since $M_{a}(G)$ is an ideal of $M(G)$, it follows from the Fubini theorem and the definition of $Q$ that

$$
\int_{X^{u}} \sum_{q \in Q} \mu_{0}\left[D+G p(E)+\sum_{1}^{u} q_{j} t_{j}\right] d t_{1} \cdots d t_{u}=0
$$

Therefore there are $u$ points $t_{1}, t_{2}, \ldots, t_{u}$ in $X$ for which the integrand in (2) is zero. Hence, in particular, we have

$$
\mu_{0}\left[D+\sum_{1}^{u} q_{j} y_{j}\right]=0 \quad(q \in Q)
$$

where $y_{j}=x_{j}+t_{j}$. Upon comparing (1) and (3), we see that if $U \subset X$ is a sufficiently small neighborhood of $0 \in G$, then the sets $U_{j}=y_{j}+U$ have the required properties.

Lemma 2. Suppose that $G$ is metrizable. Let $\mu_{0} \in M^{+}(G)$, and let $C_{0}$ be a o-compact subset of $G$ with Haar measure zero. Then there exists a strongly independent compact set $K$ in $G$ of type $M_{0}$ such that

$$
m_{G}\left[C_{0}+G p(K)\right]=\mu_{0}\left[C_{0}+G p^{\prime}(K)\right]=0 .
$$

Proof. If $q(G)$ is finite, we fix an open-and-compact subgroup $H$ of $G$ such that $\operatorname{ord}(x) \leqslant q(G)$ for all $x$ in $H$. In the other case, we set $H=G$.

Let $\left\{D_{n}\right\}_{1}^{\infty}$ be an increasing sequence of compact subsets of $G$ with $C_{0}=$ $\bigcup_{1}^{\infty} D_{n}$, and $\left\{\hat{E}_{n}\right\}_{1}^{\infty}$ a sequence of compact subsets of $\hat{G}$ with $\hat{G}=\bigcup_{1}^{\infty} \hat{E}_{n}$. We shall construct a sequence $\left\{\sigma_{n}\right\}_{1}^{\infty}$ of probability measures in $M_{0}(H)$, a sequence $\left\{I_{n}\right\}_{1}^{\infty}$ of finite collections of disjoint compact sets in $H$, a sequence $\left\{\hat{F}_{n}\right\}_{1}^{\infty}$ of compact sets in $\hat{G}$, and also a sequence $\left\{n_{p}\right\}_{1}^{\infty}$ of natural numbers. They will satisfy the following conditions (and some other conditions):

$$
\operatorname{supp} \sigma_{n} \subset \bigcup\left\{\operatorname{int}(I): I \in I_{n}\right\} \text {. }
$$




$$
\sup \left\{\widehat{\left.\sigma_{n}\right\rceil_{I}}(\chi) \mid: \chi \in \hat{G} \backslash \hat{F}_{n}\right\}<2^{-n} \sigma_{n}(I) \quad \forall I \in I_{n} .
$$

It is also assumed that each set in $I_{n+1}$ is a subset of some set in $I_{n}$.

We first take any probability measure $\sigma_{1} \in M_{0}(H)$ with compact support of diameter $<1 / 2$. Let $I$ be any compact neighborhood of supp $\sigma_{1}$ such that diam $I<$ $1, I_{1}=\{I\}$, and $n_{1}=1$. Since $\sigma_{1} \in M_{0}(G)$, we can take a compact set $\hat{F}_{1}$ in $\hat{G}$ subject to (2) with $n=1$.

Suppose that $p$ is a natural number, and that $n_{j}(1 \leqslant j \leqslant p), \sigma_{n}, I_{n}, \hat{F}_{n}$ $\left(1 \leqslant n \leqslant m=n_{p}\right)$ have been defined. Let $M_{p}$ be the largest natural number such that

$$
\max \left\{\sigma_{m}(I): I \in I_{m}\right\} \leqslant M_{p}^{-2},
$$

and write

$$
\left\{A \subset I_{m}: 1 \leqslant \text { Card } A \leqslant M_{p}\right\}=\left\{A_{r}: 1 \leqslant r \leqslant s_{p}\right\} .
$$

Setting $n_{p+1}=n_{p}+s_{p}$, we shall construct $\sigma_{n}, I_{n}$, and $\hat{F}_{n}$ for all $m<n \leqslant n_{p+1}$ as follows.

Suppose that these objects have been defined for some $n=m+r-1$ with $1 \leqslant r \leqslant s_{p}$, and put

$$
K_{n}=\left\{I \in I_{n}: I \subset J \text { for some } J \in A_{r}\right\} .
$$

Then, for each set $K$ in $K_{n}$, there are a finite collection $\left\{L_{j}^{K}\right\}_{j}$ of disjoint compact sets in $K$ and a collection $\left\{v_{j}^{K}\right\}_{j}$ of measures in $M_{0}^{+}(K)$, with supp $v_{j}^{K} \subset$ $\operatorname{int}\left(L_{j}^{K}\right)$, such that

$$
\begin{gathered}
0<\left\|v_{j}^{K}\right\|<n^{-1} \sigma_{n}(K) ; \\
\sum_{j}\left\|\nu_{j}^{K}\right\|=\sigma_{n}(K) ; \\
\left|\sum_{j}\left(\nu_{j}^{K}\right)^{\wedge}(\mathrm{x})-\left(\sigma_{n} \mid K\right)^{\wedge}(\mathrm{x})\right|<2^{-n} \sigma_{n}(K) \quad \forall \chi \in \hat{F}_{n} .
\end{gathered}
$$

To see this, it suffices to apply Lemma 3 of [11] and its obvious modification. By virtue of Lemma 1 , we can demand that the sets $L_{j}^{K}$ satisfy the following additional conditions:

$$
\begin{gathered}
\operatorname{diam} L_{j}^{K}<1 / n ; \\
0 \notin \sum_{K \in K_{n}} \sum_{j} p_{j}^{K} L_{j}^{K} \quad \forall\left(p_{j}^{K}\right) \in P_{n} ;
\end{gathered}
$$




$$
\begin{aligned}
& m_{G}\left[D_{n}+\sum_{K \in K_{n}} \sum_{j} p_{j}^{K} L_{j}^{K}\right]<2^{-n} / \operatorname{Card} P_{n} \quad \forall\left(p_{j}^{K}\right) \in P_{n} ; \\
& \mu_{0}\left[D_{n}+\sum_{K \in K_{n}} \sum_{j} q_{j}^{K} L_{j}^{K}\right]<2^{-n} / \operatorname{Card} Q_{n} \quad \forall\left(q_{j}^{K}\right) \in Q_{n} .
\end{aligned}
$$

Here $P_{n}$ is the set of all tuples $\left(p_{j}^{K}\right)$ of integers such that $\left|p_{j}^{K}\right|<q(G)$ for all $j$ and $K$ and $1 \leqslant \Sigma_{K, j}\left|p_{j}^{K}\right| \leqslant n$. Similarly $Q_{n}$ is the set of all tuples $\left(q_{j}^{K}\right)$ of integers such that $\left|q_{j}^{K}\right|=1$ for some $(K, j)$ and $\Sigma_{K, j}\left|q_{j}^{K}\right| \leqslant n$. Define

$$
\begin{gathered}
\sigma_{n+1}=\sum_{I \in K_{n}} \sigma_{n} \mid I+\sum_{K \in K_{n}} \sum_{\bar{j}} v_{j}^{K}, \\
I_{n+1}=\left(I_{n} \backslash K_{n}\right) \cup \bigcup_{K \in K_{n}}\left\{L_{j}^{K}\right\}_{j} .
\end{gathered}
$$

Then (1), with $n$ replaced by $n+1$, is satisfied. Finally we choose a compact set $\hat{F}_{n+1}$ in $\hat{G}$, with $\hat{F}_{n+1} \supset \hat{F}_{n} \cup \hat{E}_{n}$, so that (2) holds for $n+1$.

This completes our induction. It is a routine matter to prove that the sequence $\left\{\sigma_{n}\right\}_{1}^{\infty}$ converges to some probability measure $\sigma \in M_{0}(H)$ in the weak-* topology of $M(G)$, that

$$
K=\operatorname{supp} \sigma \subset \bigcap_{n=1}^{\infty} \bigcup\left\{I: I \in I_{n}\right\},
$$

and that $K$ is strongly independent. (See the proof of Lemma 4 of [11], and notice that every element of $H$ has order $\leqslant q(G)$.)

Now we want to confirm

$$
m_{G}\left[C_{0}+G p(K)\right]=\mu_{0}\left[C_{0}+G p^{\prime}(K)\right]=0 .
$$

Let $0 \neq x \in G p(K)$ be given. We have $x=\Sigma_{1}^{u} k_{i} x_{i}$ for some $\left(k_{1}, \ldots, k_{u}\right) \in$ $\mathbf{Z}^{u}$ and some distinct elements $x_{1}, \ldots, x_{u}$ of $K$. By (9), (14), and (15), there exists a natural number $N_{x}$ such that the points $x_{i}$ belong to distinct sets in $I_{n}$ whenever $n>N_{x}$. Choose any natural number $p$ so that

$$
n_{p}>N_{x}+\sum_{1}^{u}\left|k_{i}\right| \text { and } M_{p}>u \text {, }
$$

and let $A$ be the collection of all $I$ in $I_{n_{p}}$ which contain some $x_{i}(1 \leqslant i \leqslant u)$. Then $1 \leqslant \operatorname{Card} A=u<M_{p}$, and so $A \stackrel{p}{=} A_{r}$ for some $1 \leqslant r \leqslant s_{p}$ by (4). Setting $n=n_{p}+r-1$, we therefore infer from (5), (14) and (15) that $x$ belongs to the set

$$
\bigcup_{P_{n}}\left(\sum_{K \in K_{n}} \sum_{j} p_{j}^{K} L_{j}^{K}\right)
$$


Since $p$ can be chosen as large as one pleases, we conclude that

$$
G p(K) \backslash\{0\} \subset \bigcup_{n=N}^{\infty} \bigcup_{P_{n}} \sum_{K_{n}} \sum_{j} p_{j}^{K} L_{j}^{K} \quad(N=1,2, \ldots) .
$$

Similarly we have

$$
G p^{\prime}(K) \subset \bigcup_{n=N}^{\infty} \bigcup_{Q_{n}} \sum_{K_{n}} \sum_{j} q_{j}^{K} L_{j}^{K} \quad(N=1,2, \ldots) .
$$

It follows from (11) and (16) that

$$
\begin{aligned}
m_{G}\left[D_{N}+G p(K)\right] & \leqslant \sum_{n=N}^{\infty} \sum_{P_{n}} m_{G}\left[D_{N}+\sum_{K_{n}} \sum_{j} p_{j}^{K} L_{j}^{K}\right] \\
& \leqslant \sum_{n=N}^{\infty} \sum_{P_{n}} m_{G}\left[D_{n}+\sum_{K_{n}} \sum_{j} p_{j}^{K} L_{j}^{K}\right]<2^{-N+1}
\end{aligned}
$$

for all $N \geqslant 1$. (Notice that $m_{G}\left(D_{N}\right)=0$.) Letting $N \rightarrow \infty$ in (18), we have $m_{G}\left[C_{0}+G p(K)\right]=0$. Similarly we have $\mu_{0}\left[C_{0}+G p^{\prime}(K)\right]=0$ by (12) and (17). This completes the proof.

Lemma 3. Let $\mu_{0} \in M^{+}(G), C_{0}$ a $\sigma$-compact subset of $G$ which carries $\mu_{0}$, and $K$ a compact subset of $G$ such that

$$
\mu_{0}\left[C_{0}+G p^{\prime}(K)\right]=0 .
$$

Suppose that $K_{1}, K_{2}, \ldots, K_{p}$ are disjoint compact subsets of $K$ and that $\sigma_{j} \in$ $M_{c}\left(K_{j} \cup\left(-K_{j}\right)\right)$ for all $1 \leqslant j \leqslant p$.

(a) If $m=\left(m_{j}\right)_{1}^{p}$ and $n=\left(n_{j}\right)_{1}^{p}$ are different tuples of nonnegative integers, then

$$
\mu_{0} * \sigma_{1}^{m_{1}} * \cdots * \sigma_{p}^{m_{p}} \perp \mu_{0} * \sigma_{1}^{n_{1}} * \cdots * \sigma_{p}^{n_{p}}
$$

(b) If $\sigma_{j} \in M_{c}\left(K_{j}\right)$ for all $1 \leqslant j \leqslant p$ and $\nu \in L^{1}\left(\mu_{0}\right)$, then

$$
\left\|\nu * \sigma_{1}^{n_{1}} * \cdots * \sigma_{p}^{n_{p}}\right\|=\|\nu\| \cdot\left\|\sigma_{1}\right\|^{n_{1}} \cdots\left\|\sigma_{p}\right\|^{n_{p}} .
$$

Proof. To prove (a), we use the well-known method of Hewitt and Kakutani [6] (see also [10, 5.4.2]). Without loss of generality, assume that $\sigma_{j}$ $\geqslant 0$ for all $1 \leqslant j \leqslant p$ and that $m_{1}<n_{1}$. Write $\tau_{m}=\sigma_{1}^{m_{1}} * \cdots * \sigma_{p}^{m}$, and similarly for $\tau_{n}$. Putting $E_{j}=K_{j} \cup\left(-K_{j}\right)$ for $1 \leqslant j \leqslant p$, we then see that $\mu_{0} *$ $\tau_{m}$ is carried by the set $A_{m}=C_{0}+m_{1} E_{1}+\cdots+m_{p} E_{p}$. Therefore it suffices to show $\left(\mu_{0} * \tau_{n}\right)\left(A_{m}\right)=0$. Let $\lambda_{j} \in M\left(E_{j}^{n_{j}}\right)$ be the $n_{j}$-fold product of $\sigma_{j}$, and let $B_{j}$ be the set of all points $x_{j}=\left(x_{j 1}, \ldots, x_{j n_{j}}\right)$ of $E_{j}^{n_{j}}$ such that $x_{j i} \neq$ $\pm x_{j k}$ whenever $1 \leqslant i<k \leqslant n_{j}$. Since $\sigma_{j}$ is a continuous measure, we then have 
$\lambda_{j}\left(G^{n_{j}} \backslash B_{j}\right)=0$ by the Fubini theorem. On the other hand, $\left(x_{j i}\right) \in B_{1} \times \cdots \times$ $B_{p}$ implies

$$
\begin{aligned}
\mu_{0}\left[A_{m}-\sum_{j=1}^{p} \sum_{i=1}^{n_{i}} x_{j i}\right] & \leqslant \mu_{0}\left[C_{0}+m_{1} E_{1}-\sum_{i=1}^{n_{1}} x_{1 i}+\sum_{j=2}^{p} G p\left(K_{j}\right)\right] \\
& \leqslant \mu_{0}\left[C_{0}+G p^{\prime}(K)\right]=0
\end{aligned}
$$

by (*) and the definition of $G p^{\prime}(K)$. Evidently these two facts imply $\left(\mu_{0} * \tau_{n}\right)\left(A_{m}\right)=0$, as was required.

To prove (b), we need the following fact: Given $\mu \in M(G)$ and $\epsilon>0$, there is a neighborhood $V$ of $0 \in G$ such that

$$
\sigma \in M^{+}(G), \quad \operatorname{supp} \sigma-\operatorname{supp} \sigma \subset V \Rightarrow\|\mu * \sigma\| \geqslant(\|\mu\|-\epsilon)\|\sigma\| .
$$

Suppose by way of contradiction that this is false for some $\mu$ and $\epsilon$. Then, to each neighborhood $V$ of 0 there corresponds a probability measure $\sigma_{V} \in M(G)$ such that $\left\|\mu * \sigma_{V}\right\|<\|\mu\|-\epsilon$ and supp $\sigma_{V} \subset V-x_{V}$ for some $x_{V} \in G$. Upon replacing $\sigma_{V}$ by $\sigma_{V} * \delta_{x}$, we may assume that $x_{V}=0$. But then the net $\left\{\mu * \sigma_{V}\right\}$ converges to $\mu$ in the weak-* topology of $M(G)$. Hence

$$
\|\mu\| \leqslant \lim _{V} \inf \left\|\mu * \sigma_{V}\right\| \leqslant\|\mu\|-\epsilon,
$$

a contradiction.

We now prove (b) as follows. By the continuity of convolution, we can retain generality in assuming that each $\sigma_{j}$ has the form $\sigma_{j}=\Sigma_{k=1}^{q} c_{j k} \tau_{j k}$, where the $c_{j k}$ are complex numbers of absolute modulus one and the $\tau_{j k}$ are mutually singular measures in $M_{c}^{+}\left(K_{j}\right)$. Expanding $\sigma_{j}^{n_{j}}=\left(\Sigma_{k=1}^{q} c_{j k} \tau_{j k}\right)^{n_{j}}$ for all $1 \leqslant j \leqslant$ $p$ and applying part (a), we reduce (b) to the case where $\sigma_{j} \geqslant 0(1 \leqslant j \leqslant p)$, and hence to the case where $c_{j k}=1$ for all $j$ and $k$. Since we can demand that every $\tau_{j k}$ has support of sufficiently small diameter, part (b) follows from (2). This completes the proof.

Proof of Theorem B. Let $\mu \in M(G)$, and $\Phi$ a separable subset of $L^{1}(\mu)$. Given $\sigma \in M(G)$, we let $\sigma_{s}$ denote the singular part of $\sigma$ with respect to $m_{G}$. Notice that

$$
r(\sigma)=\lim _{n \rightarrow \infty}\left\|\sigma^{n}+M_{a}(G)\right\|^{1 / n}=\lim _{n \rightarrow \infty}\left\|\left(\sigma^{n}\right)_{s}\right\|^{1 / n}
$$

since $M_{a}(G)$ is an ideal in $M(G)$ with spectrum $\hat{G}$. Now define $\mu_{0}$ to be the singular part of $\exp (|\mu|)$, and choose a $\sigma$-compact subset $C_{0}$ of $G$ so that $m_{G}\left(C_{0}\right)=$ $\mu_{0}\left(G \backslash C_{0}\right)=0$. Then $\nu \in L^{1}(\mu)$ implies $\left(\nu^{n}\right)_{s} \in L^{1}\left(\mu_{0}\right)$ for all $n \in Z^{+}$.

We first assume that $G$ is metrizable, and take a compact subset $K$ of $G$ as 
where $H=H_{\Gamma}$ is the annihilator of $\Gamma$ in $G$ and $m_{H}$ denotes the Haar measure of $H$ of norm one. This can be proved by considering the Fourier transform of $\nu$ and by applying Theorem 1.9.1 of [10]. Since $\Phi \subset L^{1}(\mu)$ is separable, there is a $\sigma$-compact open subgroup $\Gamma$ of $G$ such that

$$
\left\|\left(\nu^{n}\right)_{s}\right\|=\left\|\left(\nu^{n}\right)_{s} * m_{H}\right\| \quad \forall \nu \in \Phi \text { and } \forall n \in \mathbf{Z}^{+} .
$$

By Lemma 6 of [11], we may assume that $G_{0}=G / H$ is metrizable and $m_{G}\left(C_{0}+H\right)=0$. Let $\pi: G \rightarrow G_{0}$ be the natural quotient map, and let

$$
\nu \rightarrow \pi^{*}(\nu)=\nu \circ \pi^{-1}: M(G) \rightarrow M\left(G_{0}\right)
$$

be the measure algebra homomorphism induced by $\pi$. Then it is easy to check that $\pi^{*}$ maps $M_{a}^{+}(G)$ onto $M_{a}^{+}\left(G_{0}\right), M_{0}^{+}(G)$ onto $M_{0}^{+}\left(G_{0}\right)$, and $L^{1}\left(\mu_{0}\right)$ onto $L^{1}\left(\pi^{*}\left(\mu_{0}\right)\right)$ (cf. $\left.[14,2.2 .4]\right)$. Moreover, we have $\left\|\pi^{*}(\nu)\right\|=\left\|\nu * m_{H}\right\|$ for all $\nu \in M(G)$, as is easily seen. It follows from (9) that

$$
\left\|\pi^{*}\left[\left(\nu^{n}\right)_{s}\right]\right\|=\left\|\left(\nu^{n}\right)_{s}\right\| \quad \forall n \in \mathbf{Z}^{+}
$$

for all $\nu \in \Phi$. Obviously (10) is satisfied for every $\nu \in M^{+}(G)$ as well.

Since $m_{G_{0}}\left[\pi\left(C_{0}\right)\right]=m_{G}\left(C_{0}+H\right)=0$ and $\pi^{*}\left(\mu_{0}\right)$ is carried by the set $\pi\left(C_{0}\right)$, we have $L^{1}\left(\pi^{*}\left(\mu_{0}\right)\right) \subset M_{s}\left(G_{0}\right)$. In particular $\pi^{*}\left[\left(\nu^{n}\right)_{s}\right]$ is the singular part of $\left(\pi^{*}(\nu)\right)^{n}=\pi^{*}\left(\nu^{n}\right)$ for every $\nu \in L^{1}(\mu)$ and every $n \in Z^{+}$. Hence $r\left[\pi^{*}(\nu)\right]=r(\nu)$ for all $\nu \in\left[L^{1}(\mu) \cap M^{+}(G)\right] \cup \Phi$, by (10). To complete the proof, it therefore suffices to note that $\pi^{*}\left[M_{0}^{+}(G)\right]=M_{0}^{+}\left(G_{0}\right)$, that $\pi^{*}[M(G)]$ $=M\left(G_{0}\right)$, and that the adjoint map of $\pi^{*}$ sends $\Delta_{G_{0}} \backslash \Sigma_{G_{0}}$ into $\Delta_{G} \backslash \Sigma_{G}$ in a one-to-one way. This establishes Theorem $B$ for all nondiscrete groups.

Proof of Corollary 1. Let $Y \subset \Delta_{0}$ be a boundary of $M_{0}(G)$, and $\mu \in M(G)$. Choose any $f \in \Delta_{G}$ such that $|\hat{\mu}(f)|=\|\hat{\mu}\|_{\Delta_{G}}$. If $f \in \hat{G}$, we take $\lambda \in M_{a}(G)$ so that $0 \leqslant \hat{\lambda} \leqslant 1$ on $\hat{G}$ and $\hat{\lambda}(f)=1$. Then we have $\lambda * \mu \in$ $M_{0}(G)$ and $\|\hat{\lambda * \mu}\|_{\Delta_{G}}=|\hat{\mu}(f)|$; hence $|\hat{\mu}(g)|=|\hat{\lambda} * \mu(g)|=|\hat{\mu}(f)|$ for some $g \in Y$. If $f \notin \hat{G}$, then $r(\mu)=|\hat{\mu}(f)|$. By Theorem B, we can find a probability measure $\tau \in M_{0}(G)$ such that $r(\tau * \mu)=r(\mu)$. Then $|\hat{\mu}(g)|=|\tau * \mu(g)|=$ $r(\mu)=|\hat{\mu}(f)|$ for some $g \in Y$, which establishes part (a).

To prove (b), first notice that $\overline{\Delta_{G} \backslash \Sigma_{G}} \subset \Delta_{G} \backslash \hat{G}$ since $\hat{G}$ is open and is contained in $\Sigma_{G}$. If the above two sets were different, there would exist a nonempty open set $U$ in $\Delta_{G}$ such that $U \cap \overline{\Delta_{G} \backslash \Sigma_{G}}=\varnothing \neq U \backslash \hat{G}$. Since the space of all Gelfand transforms of measures is closed under the complex conjugation on $\Sigma_{G}$, it would follow from the Stone-Weierstrass theorem that there would exist a $\hat{\mu} \in$ $M(G)$ such that $0 \leqslant \hat{\mu} \leqslant 1$ on $\Sigma_{G}, \hat{\mu}(f)=1$ for some $f \in U \backslash \hat{G}$, and $\hat{\mu}<1 / 2$ on $\Sigma_{G} \backslash U$. Then the set $U \cap \hat{\mu}^{-1}(1)$ would be a local peak set for $M(G)$, and therefore would be a peak set for $M(G)$ by Rossi's theorem [4]. Consequently 
in Lemma 2. Let $\sigma_{1}, \sigma_{2}, \ldots, \sigma_{p}$ be mutually singular measures in $M_{c}(K)$, and let $z_{1}, z_{2}, \ldots, z_{p}$ be complex numbers satisfying $\left|z_{j}\right| \leqslant\left\|\sigma_{j}\right\|(1 \leqslant j \leqslant p)$. We then claim that given $\nu \in L^{1}(\mu)$ there exists an element $f$ in $\Delta_{G} \backslash \hat{G}$ such that

$$
|f(\nu)|=r(\nu) \text { and } f\left(\sigma_{j}\right)=z_{j} \quad(1 \leqslant j \leqslant p) .
$$

There is no loss of generality in assuming $\left\|\sigma_{j}\right\|=1$ for all $j$. Let $\tau_{2 j-1}$ and $\tau_{2 j}$ be mutually singular measures in $L^{1}\left(\sigma_{j}\right)$ such that $\sigma_{j}=\left(\tau_{2 j-1}+\tau_{2 j}\right) / 2$ and $\left\|\tau_{2 j-1}\right\|=\left\|\tau_{2 j}\right\|=1$, and write $z_{j}=\left(w_{2 j-1}+w_{2 j}\right) / 2$ with $\left|w_{2 j-1}\right|=\left|w_{2 j}\right|=$ 1. Since $m_{G}\left[C_{0}+G p(K)\right]=0$, it follows from Lemma 3 that

$$
\begin{aligned}
\left\|\left[\nu *\left(\delta_{0}+\sum_{k=1}^{2 p} \bar{w}_{k} \tau_{k}\right)\right]^{n}+M_{a}(G)\right\| \\
=\left\|\left(\nu^{n}\right)_{s} *\left(\delta_{0}+\sum_{k=1}^{2 p} \bar{w}_{k} \tau_{k}\right)^{n}\right\| \\
=\left\|\left(\nu^{n}\right)_{s}\right\|\left(1+\sum_{k=1}^{2 p}\left\|\tau_{k}\right\|\right)^{n}=\left\|\left(\nu^{n}\right)_{s}\right\|(1+2 p)^{n},
\end{aligned}
$$

which yields

$$
r\left[\nu *\left(\delta_{0}+\sum_{k=1}^{2 p} \bar{w}_{k} \tau_{k}\right)\right]=r(\nu) \cdot(1+2 p) .
$$

We can therefore find an element $f \in \Delta_{G} \backslash \hat{G}$ such that

$$
|f(\nu)|=r(\nu) \text { and } f\left(\tau_{k}\right)=w_{k} \quad(1 \leqslant k \leqslant 2 p) .
$$

By the choices of $\tau_{k}$ and $w_{k},(1)^{\prime}$ implies (1), which establishes our claim.

We next assert that, given $\nu \in L^{1}(\mu)$, every linear functional on $M_{c}(K)$, of norm $\leqslant 1$, extends to an element $f \in \Delta_{G} \mid \hat{G}$ such that $|f(\nu)|=r(\nu)$. In fact, this is an easy consequence of (1) and the arguments of Hewitt and Kakutani in [6]. We leave the details to the reader.

Now choose three disjoint compact sets $K_{j}$ in $K(j=1,2,3)$, each of type $M_{0}$, and fix two probability measures $\lambda \in M_{0}\left(K_{1}\right)$ and $\tau \in M_{0}\left(K_{2}\right)$. We now prove that $\tau$ and the set

$$
X=\left\{f \in \Delta_{G}: f(\lambda)=1,\left|f\left(\lambda^{*}\right)\right| \leqslant 1 / 2\right\} \cup\left\{f \in \Delta_{G}:\left|1-f\left(\lambda * \lambda^{*}\right)\right| \geqslant 3 / 2\right\}
$$

have the required property. It is obvious that $X$ is a compact subset of $\Delta_{0} \backslash \Sigma_{G}$. Let $c$ be a complex number of modulus $\leqslant 1$, and $\nu \in L^{1}(\mu)$. Let also $\varphi$ be an arbitrary (linear) functional on $M_{c}\left(K_{3}\right)$, of norm $\leqslant 1$. By the Hahn-Banach theorem, $\varphi$ extends to a functional $\psi$ on $M_{c}(K)$, of norm one, such that $\psi(\lambda)=1$ and $\psi(\tau)=c$. It follows from the result asserted in the last paragraph that there 
is an $f$ in $\Delta_{G} \backslash \hat{G}$ such that $|f(\nu)|=r(\nu), f(\lambda)=1, f(\tau)=c$ and $f=\varphi$ on $M_{c}\left(K_{3}\right)$. We want to show that such an $f$ can be chosen from the set $X$. If $\left|f\left(\lambda^{*}\right)\right|$ is less than $1 / 2$, then there is nothing to prove; so assume $\left|f\left(\lambda^{*}\right)\right| \geqslant$ $1 / 2$. Setting $\tau_{1}=\lambda * \lambda^{*}$, we then have

$$
\left\|\left(\nu^{m}\right)_{s} * \tau_{1}^{n}\right\| \geqslant\left|f\left(\nu^{m} * \tau_{1}^{n}\right)\right| \geqslant r(\nu)^{m}(1 / 2)^{n}
$$

for all $m$ and $n \in \mathbf{Z}^{+}$, so that

$$
r\left[\nu^{m} *\left(\delta_{0}-\tau_{1}\right)\right] \geqslant r(\nu)^{m}(3 / 2) \quad\left(m \in \mathbf{Z}^{+}\right)
$$

by (*) and Lemma 3. Putting $\mu_{1}=\mu_{0} * \exp \left(\tau_{1}\right)$, we also see that $\mu_{1}$ is carried by the $\sigma$-compact set $C_{1}=C_{0}+G p\left(K_{1}\right)$ and that

$$
\begin{aligned}
\mu_{1}\left[C_{1}+G p^{\prime}\left(K_{2} \cup K_{3}\right)\right] & =\int \mu_{0}\left[C_{1}+G p^{\prime}\left(K_{2} \cup K_{3}\right)-y\right] d \theta(y) \\
& \leqslant \mu_{0}\left[C_{0}+G p^{\prime}(K)\right] \cdot e=0,
\end{aligned}
$$

where $\theta=\exp \left(\tau_{1}\right)$. Therefore, if $\tau_{2}, \ldots, \tau_{p}$ are mutually singular probability measures in $M_{c}\left(K_{2} \cup K_{3}\right)$ and if $m, n, n_{2}, \ldots, n_{p} \in \mathrm{Z}^{+}$, then

(5) $\left\|\left[\nu^{m} *\left(\delta_{0}-\tau_{1}\right)\right]^{n} * \tau_{2}^{n}{ }^{n} * \cdots * \tau_{p}^{n} p+M_{a}(G)\right\| \geqslant r\left[\nu^{m} *\left(\delta_{0}-\tau_{1}\right)\right]^{n}$

by Lemma 3 (applied to $\mu_{1}$ and $C_{1}$ ). Consequently, one more application of Lemma 3, combined with (5), yields

$$
r\left[\nu^{m} *\left(\delta_{0}-\tau_{1}\right) *\left(\delta_{0}+\sum_{j=2}^{p} \bar{z}_{j} \tau_{j}\right)\right]=r\left[\nu^{m} *\left(\delta_{0}-\tau_{1}\right)\right] \cdot p
$$

for all complex numbers $z_{2}, \ldots, z_{p}$ of absolute modulus one. (Notice that the left-hand side of (6) cannot be larger than the right-hand one.) Therefore there is a $g_{m} \in \Delta_{G} \backslash \hat{G}$ such that

$$
\left|g_{m}\left[\nu^{m} *\left(\delta_{0}-\tau_{1}\right)\right]\right|=r\left[\nu^{m} *\left(\delta_{0}-\tau_{1}\right)\right], \quad g_{m}\left(\tau_{j}\right)=z_{j} \quad(2 \leqslant j \leqslant p) .
$$

It follows from (4) and the first equality of (7) that $\left|1-g_{m}\left(\tau_{1}\right)\right| \geqslant 3 / 2$, and so $g_{m} \in X$; moreover $\left|g_{m}(\nu)\right|=\left|g_{m}\left(\nu^{m}\right)\right|^{1 / m} \geqslant r(\nu)(3 / 4)^{1 / m}$ by (7) and (4). Recalling that $X$ is compact and letting $m \rightarrow \infty$, we find an element $h \in X$ such that

$$
|h(\nu)|=r(\nu) \text { and } h\left(\tau_{j}\right)=z_{j} \quad(2 \leqslant j \leqslant p) .
$$

We repeat almost the same argument as before to obtain an $f \in X$ with the required property. Since it is easy to prove that the conjugate space of $M_{c}\left(K_{3}\right)$ has cardinality equal to $2^{c}$, this establishes Theorem $B$ for metrizable groups.

The proof for the nonmetrizable case is now easy. We first note that given $\nu \in M(G)$ there is a $\sigma$-compact open subgroup $\Gamma$ of $\hat{G}$ such that $\left\|\nu * m_{H}\right\|=\|\nu\|$, 
there would exist a $\nu \in M(G)$ such that $\hat{v}(f)=1,|\hat{v}| \leqslant 1$ on $\Delta_{G}$, and $|\hat{v}|<$ $1 / 2$ on $\Delta_{G} \backslash \Sigma_{G}$. But then $r(v)=1$, which contradicts Theorem B. This establishes part (b).

By Theorem B, no element of $\Delta_{G} \backslash \hat{G}$ can be a peak point for $M(G)$; hence $P_{G} \subset \hat{G}$. Therefore part (c) is an easy consequence of the fact that $G$ is $\sigma$-compact if and only if $\hat{G}$ is metrizable [7]. This completes the proof.

Proof of Corollary 2. Let $\mu \in M(G)$ be given. Notice that $\Delta_{G} \backslash \hat{G}$ is the spectrum of the quotient algebra $M(G) / M_{a}(G)$. Choose a countable dense subset $D$ of $C \backslash \hat{\mu}\left(\Delta_{G} \backslash \hat{G}\right)$. For each $c \in D$, there is a $\nu_{c} \in M(G)$ such that $\hat{\nu}_{c}=$ $(c-\hat{\mu})^{-1}$ on $\Delta_{G} \backslash \hat{G}$. Setting $\Phi=\left\{\nu_{c}: c \in D\right\}$, we apply Theorem B to find a compact set $X$ in $\Delta_{0} \backslash \Sigma_{G}$ such that

$$
\sup \left\{|c-\hat{\mu}(f)|^{-1}: \hat{f} \in X\right\}=\sup \left\{|c-\hat{\mu}(g)|^{-1}: g \in \Delta_{G} \backslash \hat{G}\right\}
$$

for all $c \in D$. Since $\hat{\mu}(X)$ is compact, this implies that $\hat{\mu}(X)$ contains all the boundary points of $\hat{\mu}\left(\Delta_{G} \backslash \hat{G}\right)$ in $\mathbf{C}$.

If Card $\left[\hat{\mu}\left(\Delta_{0} \backslash \Sigma_{G}\right)\right]<c$, then $\dot{\mu}\left(\Delta_{G} \backslash \hat{G}\right)$ has a countable boundary since it is compact. Therefore $\hat{\mu}\left(\Delta_{G} \backslash \hat{G}\right)$ itself is countable, so that $\hat{\mu}\left(\Delta_{G} \backslash \hat{G}\right)=$ $\hat{\mu}\left(\Delta_{0} \backslash \Sigma_{G}\right)$ by the result already established. If $\hat{\mu}$ is real on $\Delta_{0} \backslash \Sigma_{G}$, then $\hat{\mu}$ must be real on $\Delta_{G} \backslash \hat{G}$, hence $\hat{\mu}\left(\Delta_{G} \backslash \hat{G}\right)$ has no interior point, and hence $\hat{\mu}\left(\Delta_{G} \mid \hat{G}\right)=$ $\hat{\mu}\left(\Delta_{0} \backslash \Sigma_{G}\right)$. This establishes Corollary 2 .

REMARKS. (a) Theorem A implies $\hat{\mu}_{d}\left(\Delta_{G}\right) \subset \hat{\mu}\left(\Delta_{0} \backslash \Sigma_{G}\right)$ for all $\mu \in M(G)$. Moreover, we can prove that $\hat{\mu}_{d}\left(\Delta_{G}\right) \subset \hat{\mu}\left(\Delta_{0} \cap \Sigma_{G} \backslash \hat{G}\right)$ by applying the methods in [11].

(b) Notice that $\delta_{0}\left(C_{1}+C_{2}\right)=0$ if and only if $C_{1} \cap\left(-C_{2}\right)$ is empty. If we only require that $C_{0} \cap G p^{\prime}(K)=\varnothing$ in Lemma 2 instead of that $m_{G}\left[C_{0}+G p(K)\right]=\mu_{0}\left[C_{0}+G p^{\prime}(K)\right]=0$, then the assumption that $C_{0}$ is a $\sigma$ compact set with $m_{G}\left(C_{0}\right)=0$ can be weakened to be that $C_{0}$ is a set of the first category in $G$ (cf. $[5,2.1]$ ).

(c) In some special cases, the proof of Theorem B can be somewhat simpli. fied and we have a result slightly stronger than Theorem $B$.

Let $H_{0}$ be an open subgroup of $G$ of the form $H_{0}=\mathrm{R}^{n} \times H_{1}$, where $n$ is a nonnegative integer and $H_{1}$ is a compact subgroup of $G$ (cf. [10, 2.4.1]). Let $P$ be the set of all $p \in Z$ such that $1 \leqslant p<q\left(H_{1}\right)$ and $\operatorname{Card}\left\{\chi \in \hat{H}_{1}: \chi^{p}=1\right\}<$ $\infty$. Then the last condition in Lemma 2 can be strengthened to be that $m_{G}\left[C_{0}+G p(K)\right]=\mu_{0}\left[C_{0}+K(P)\right]=0$. Here $K(P)$ denotes the set of all points $x$ of the form $x=\Sigma_{1}^{u} k_{j} x_{j}$, where $u=u_{x}$ is a natural number, $x_{1}, x_{2}, \ldots, x_{u}$ are distinct elements of $K$, and $k_{1}, k_{2}, \ldots, k_{u}$ are integers such that $\left|k_{j}\right| \in P$ for some $1 \leqslant j \leqslant u$. The case where $2 \in P$ is particularly interesting.

Suppose in Lemma 3 that $\mu_{0}, C_{0}$ and $K$ are such that $\mu_{0}\left[C_{0}+K(\{1,2\})\right]$ $=0$. Then we can prove that 


$$
\left\|\nu * \sigma_{1}^{n_{1}} * \cdots * \sigma_{p}^{n_{p}}\right\|=\|\nu\| \cdot\left\|\sigma_{1}\right\|^{n_{1}} \cdots\left\|\sigma_{p}\right\|^{n_{p}}
$$

for all $\nu \in L^{1}\left(\mu_{0}\right)$ and all $\sigma_{j} \in M_{c}\left(K_{j} \cup\left(-K_{j}\right)\right)$. Therefore a moment's glance at the proof of Theorem B yields this result: If either $q(G)=2$, or $G$ contains an open subgroup $H_{0}$ as above with $2 \in P$, then the measure $\tau$ in Theorem B can be taken so that $\tau=\lambda * \lambda^{*}$ for some $\lambda \in M_{0}^{+}(G)$. We omit the details.

(d) If $\mu \in M^{+}(G)$, then the number $r(\mu)$ is in $\hat{\mu}\left(\Delta_{0} \backslash \Sigma_{G}\right)$. To see this, choose a complex number $z$ of absolute modulus one so that $z r(\mu) \in \hat{\mu}\left(\Delta_{G} \backslash \hat{G}\right)$. Then we have

$$
\begin{aligned}
r\left(\delta_{0}+\mu\right) & =\lim _{n \rightarrow \infty}\left\|\left[\left(\delta_{0}+\mu\right)^{n}\right]_{s}\right\|^{1 / n} \\
& \geqslant \lim _{n \rightarrow \infty}\left\|\left[\left(\delta_{0}+\bar{z} \mu\right)^{n}\right]_{s}\right\|^{1 / n}=1+r(\mu),
\end{aligned}
$$

and so $r\left(\delta_{0}+\mu\right)=1+r(\mu)$. Thus our assertion follows from Theorem B with $\Phi=\left\{\delta_{0}+\mu\right\}$.

(e) Let $M_{0}^{\infty}(G)$ denote the $L$-ideal in $M(G)$ generated by all measures $\mu$ of the form $\mu=\mu_{1} * \mu_{2} * \cdots$, where the $\mu_{j}$ are probability measures in $M_{0}(G)$ and the infinite convolution product is assumed to converge in the weak-* topology of $M(G)$. Let also $\Delta_{0}^{\infty}$ denote the spectrum of $M_{0}(G)$ identified with an open subset of $\Delta_{G}$. Then it is not difficult to prove that $\operatorname{Card}\left(\Delta_{0} \backslash \Delta_{0}^{\infty}\right) \geqslant 2^{c}$. Moreover, in Theorem B, we can replace $M_{0}(G)$ and $\Delta_{0}$ by $M_{0}^{\infty}(G)$ and $\Delta_{0}^{\infty}$, respectively. Using this result, we can prove that if $Y$ is a boundary of $M_{0}^{\infty}(G)$, then $\left(Y \backslash \Sigma_{G}\right) \cup(Y \cap \hat{G})$ is a boundary of $M(G)$, which of course improves part (a) of Corollary 1. Similary the set $\Delta_{0}$ in Corollary 2 can be replaced by $\Delta_{0}^{\infty}$.

(f) Finally we list three problems which the author has been unable to solve.

(i) Is it true that $\hat{\mu}\left(\Delta_{G} \backslash \hat{G}\right)=\hat{\mu}\left(\Delta_{0} \backslash \Sigma_{G}\right)$ for all $\mu \in M(G)$ ?

(ii) Does $\hat{\mu}\left(\Sigma_{G} \backslash \hat{G}\right)=\{0\}$ imply $r(\mu)=0$ ?

(iii) Does $\Sigma_{G} \backslash \hat{G}$ contain any strong boundary point for $M(G)$ ?

\section{REFERENCES}

1. G. Brown, $M_{0}(G)$ has a symmetric maximal ideal off the Šilov boundary, Proc. London Math. Soc. (3) 27 (1973), 484-504. MR 48 \#2666.

2. G. Brown and W. Moran, $L^{1 / 2}(G)$ is the kernel of the asymmetric maximal ideals of $M(G)$, Bull. London Math. Soc. 5 (1973), 179-186. MR 49 \#3450. 350-368. MR 50 \#14060.

3. -,$M_{0}(G)$-boundaries are $M(G)$-boundaries, J. Functional Analysis 18 (1975),

4. T. W. Gamelin, Uniform algebras, Prentice-Hall, New York, 1969.

5. C. C. Graham, Measures vanishing off the symmetric maximal ideals of $M(G)$, Proc. Cambridge Philos. Soc. 75 (1974), 51-61. MR 49 \#9539.

6. E. Hewitt and S. Kakutani, A class of multiplicative linear functionals on the 
measure algebra of a locally compact Abelian group, Illinois J. Math. 4 (1960), 553-574. MR 23 \#A527.

7. E. Hewitt and K. R. Stromberg, A remark on Fourier-Stieltjes transforms, An. Acad. Brasil. Ci. 34 (1962), 175-180. MR 27 \#533.

8. K. Izuchi and T. Shimizu, Topologies on groups and a certain L-ideal of measure algebras, Tôhoku Math. J. 25 (1973), 53-60.

9. L.-A. Lindahl and F. Poulsen, Thin sets in harmonic analysis, Dekker, New York, 1971.

10. W. Rudin, Fourier analysis on groups, Interscience Tracts in Pure and Appl. Math., no. 12, Interscience, New York and London, 1962. MR 27 \#2808.

11. S. Saeki, Symmetric maximal ideals in $M(G)$, Pacific J. Math. 53 (1974), 229-243.

12. K. Saka, $A$ note on subalgebras of a measure algebra vanishing on non-symmetric homomorphisms, Tôhoku Math. J. (2) 25 (1973), 333-338. MR 50 \#10686.

13. T. Shimizu, L-ideals of measure algebras, Proc. Japan Acad. 48 (1972), 172-176. MR 47 \#326.

14. J. L. Taylor, Measure algebras, CBMS Regional Conf. Ser. in Math., no. 16, Amer. Math. Soc., Providence, R. I., 1973.

15. J. H. Williamson, Banach algebra elements with independent powers and theorems of Wiener-Pitt type, Function Algebras (Proc. Internat. Sympos. on Function Algebras, Tulane Univ., 1965), Scott, Foresman, Chicago, Ill., 1966, pp. 186-197. MR 33 \#6302.

DEPARTMENT OF MATHEMATICS, TOKYO METROPOLITAN UNIVERSITY, SETAGAYA, TOKYO, JAPAN 